\title{
Suicidal Antidepressant Overdoses: A Comparative Analysis by Antidepressant Type
}

\author{
Nicole C. White, $B A^{a}$, Toby Litovitz, $M D^{b}$, Cathleen Clancy, $M D^{b, c}$ \\ aUniversity of Virginia School of Medicine, Charlottesville, VA \\ bNational Capital Poison Center, Washington, DC \\ cThe George Washington University Department of Emergency Medicine, Washington, DC
}

\begin{abstract}
Introduction: The safety of antidepressants following overdose is critical because of the high risk of suicide attempts in depressed patients. This study was conducted to decrease the fatality rate of antidepressant overdoses by providing data to shift prescribing toward safer antidepressants.

Methods: US poison control data for 2000-2004 were analyzed by 25 antidepressant types. Medical outcome differences were quantified using a hazard index (number of major or fatal outcomes per 1000 reported antidepressant ingestions).

Results: Of 82,802 suicidal single-agent ingestions of identifiable antidepressants approved for use in the US, cases occurred predominantly in females and peaked in teens. Fatal cases peaked at 40 to 49 years of age. Suicidal ingestions of the SSRIs, SNRIs, and other antidepressants peaked in teens, lithium in the twenties, tricyclics and tetracyclics in the thirties, and MAO inhibitors in the forties. There were 40 major or fatal outcomes per 1000 cases. Weighted by antidepressant type, the mean hazard index for the 25 antidepressants was 79 (range: 0 to 292). Amoxapine (292), maprotiline (211), and desipramine (187) had the highest hazard indices. The tricyclic antidepressants, MAO inhibitors, maprotiline, and bupropion were in the more severe half of antidepressants, ranked by hazard index. All SSRIs had low hazard indices. Hazard index and exposure frequency were inversely correlated $(\mathrm{R}=-0.423$, $\mathrm{p}=$ 0.035), while hazard index and use of critical care were positively correlated for the 25 antidepressant types $(\mathrm{R}=0.797, \mathrm{p}<0.001)$. Clinical effect profiles for each antidepressant type are presented.
\end{abstract}

Conclusion: Suicidal overdose severity varied considerably by antidepressant type. Prescribing decisions should be informed by regularly updated comparative overdose severity data.

\section{INTRODUCTION}

It has been estimated that a quarter of patients diagnosed with major depression attempt suicide in a lifetime, and 15\% of those patients ultimately die from suicide [1]. Antidepressants have been shown to be highly effective treatments for depression; paradoxically, to achieve compliance, the prescriber must supply a toxic drug, giving the patient ready access to a suicidal method. Complicating the paradox are recently mandated blackbox warnings on all antidepressants warning of an increased risk of suicidal symptoms in children, adolescents and young adults treated with these medications, while simultaneously acknowledging that failing to treat the underlying disease also carries a risk of suicidal behavior [2]. Given the high risk of suicide in depressed patients, the overdose safety of antidepressants is of great significance from both clinical and public health perspectives.

The impulsivity that often characterizes suicides provides the rationale for means restriction-the concept that restricting access to lethal methods during this transient period of ambivalence may

Keywords: antidepressant overdose; antidepressant toxicity; poisoning hazard

Note: There was no outside funding of any kind used for this study.

Corresponding Author: Toby Litovitz, MD, National Capital Poison Center, 3201 New Mexico Avenue, Suite 310, Washington, DC 20016. Email: toby@poison.org 
reduce suicide rates [3]. Many studies suggest that methods of means restriction-including gun-control legislation, pesticide restrictions, domestic gas detoxification (switching to natural gas), barbiturate restrictions, analgesic packaging changes, catalytic converters, and barriers to jumping-simply drove suicidal individuals to other methods, thereby maintaining the overall suicide rate $[4,5,6,7,8]$. Still, several isolated studies found no indication of means substitution, one following gun-control legislation in Canada [9] and the other following the introduction of catalytic converters in England [10]. Interviews with survivors of suicidal falls from the Golden Gate Bridge suggest that some subjects may have only one preferred method of suicide; in such cases, method restriction could prevent suicide [5]. While suicidal patients may eventually seek alternative methods of suicide, reduced prescribing of the more toxic antidepressants would decrease their availability and reduce suicides from antidepressant overdose.

Since the introduction of psychoactive substances in the 1950s, overdoses appear to parallel sales for each drug [11]. Such opportunistic overdoses call for shifting prescribing patterns toward less toxic drugs that are equally efficacious. This may not reduce the number of overdoses, but could be expected to reduce fatality rates.

This study used poison control data to investigate relative antidepressant overdose severity. US poison center data are compiled in the Toxic Exposure Surveillance System (TESS) by the American Association of Poison Control Centers (AAPCC) [12]. TESS was renamed the National Poison Data System (NPDS) in 2007. The name TESS is used throughout here, however, reflecting the database name when this study was conducted. Of all exposures reported to TESS, $8.0 \%$ are coded "suspected suicidal," or "resulting from the inappropriate use of a substance for reasons that are suspected to be self-destructive or manipulative." Suicidal cases are among the most serious cases reported to poison centers, accounting for $54.0 \%$ of the poisoning fatalities reported in 2004, but comprising only $8.0 \%$ of all human poison exposures reported to TESS.

This study was conducted to decrease the fatality rate of antidepressant overdoses by providing practitioners with updated data quantifying safety differences between all the antidepressants currently marketed in the US. Awareness of these safety comparisons and overdose profiles could effectively shift prescribing practices toward safer antidepressants.

\section{METHODS}

TESS data for human, single-substance, antidepressant ingestions were analyzed for the 5-year period, 2000 through 2004 [Appendix A]. Duplicate cases reported to more than one poison center and confirmed nonexposures were excluded (where coded as such in TESS).

All cases categorized as suicidal ingestions of an antidepressant categorized in one of the following AAPCC generic codes were reviewed: amitriptyline, amoxapine, desipramine, doxepin, imipramine, maprotiline, nortriptyline, protriptyline, other cyclic antidepressant, unknown cyclic antidepressant, lithium, monoamine oxidase (MAO) inhibitor, selective serotonin reuptake inhibitor (SSRI), trazodone, other antidepressant, and unknown antidepressant. Cases with antidepressants formulated with a benzodiazepine, phenothiazine, or other antipsychotic were excluded, as were cases involving antidepressants formulated for topical use.

The 988 specific pharmaceutical products implicated in cases categorized as exposures to the AAPCC antidepressant generic codes listed above were reviewed for categorization accuracy. All specific products were recategorized using the more specific categorization schema outlined in Table 1.

A number of TESS fields enable characterization of the severity of a poison exposure, including medical outcome, management site and level of care, specific clinical effects observed, and treatments provided [13]. Ten options are provided for coding medical outcome, including 5 definitive outcomes in which adequate follow-up is obtained to accurately categorize the outcome [12]:

\section{Definitive outcomes}

No effect: No signs or symptoms resulted from the exposure.

Minor effect: Minimally bothersome signs and symptoms resulted from the exposure, but these resolved rapidly without residual disability or disfigurement.

Moderate effect: More pronounced, prolonged or systemic signs and symptoms developed compared to patients with a minor effect. These patients usually require treatment, but the effects are not life-threatening and there is no residual disability.

Major effect: Life-threatening signs or symptoms developed as a result of the exposure, or the patient manifested significant residual disability or disfigurement.

Death: A fatality occurred as a direct result of the poison exposure or as a complication of the exposure.

Other outcomes are defined elsewhere, including: not followed, nontoxic exposure; not followed, minimal clinical effects possible; unable to follow, potentially toxic exposure; unrelated effect; and confirmed nonexposure.

To quantify the difference in medical outcome by antidepressant types, a hazard index was calculated as the number of cases with a major or fatal outcome per 1000 single-agent ingestions of that antidepressant type.

TESS data also include documentation of the occurrence of 131 different clinical effects grouped in 9 categories. Each clinical effect is coded as "related," "unrelated," or "unknown if related." The distribution of clinical effects associated with each antidepressant type was characterized, focusing on those effects documented as "related" or "unknown if related." For cases managed in a healthcare facility, the level of care provided is further qualified in TESS data as: treated and released, admitted to critical care, admitted to noncritical care, admitted to a psychiatric facility, refused referral, or lost to follow-up. The percentage of 
patients requiring critical care was compared across the antidepressant types.

Data were compiled in an SQL database and analyzed using the TESS software. Graphs and tables were prepared in Excel. Chi squares, the significance of correlation coefficients, and T-tests were determined using SISA Binomial [14].

\section{RESULTS}

From 2000 to 2004 , 28\% of suicidal ingestions reported to US poison centers involved antidepressants. During the 5-year period, suicidal ingestions were implicated in 898,751 TESS cases.

Of these, 253,601 involved antidepressants, and 84,670 involved

Table 1: Outcome Distribution and Critical Care Utilization by Antidepressant Type

\begin{tabular}{|c|c|c|c|c|c|}
\hline & No. of Cases & $\begin{array}{l}\text { Critical } \\
\text { Care (\%) }\end{array}$ & $\begin{array}{c}\text { Major } \\
\text { Outcome (No.) }\end{array}$ & $\begin{array}{c}\text { Fatal } \\
\text { Outcome (No.) }\end{array}$ & Hazard Index \\
\hline \multicolumn{6}{|l|}{ Tricyclics Antidepressants } \\
\hline Amoxapine & 24 & 50.0 & 5 & 2 & 292 \\
\hline Desipramine & 187 & 56.7 & 29 & 6 & 187 \\
\hline Amitriptyline & 8,316 & 54.2 & 1,220 & 61 & 154 \\
\hline Doxepin & 1,758 & 50.2 & 242 & 19 & 148 \\
\hline Imipramine & 700 & 50.3 & 82 & 13 & 136 \\
\hline Nortriptyline & 996 & 44.1 & 85 & 3 & 88 \\
\hline Clomipramine & 190 & 41.1 & 13 & 1 & 74 \\
\hline Protriptyline & 16 & 56.3 & 1 & 0 & 63 \\
\hline Trimipramine & 18 & 55.6 & 1 & 0 & 56 \\
\hline \multicolumn{6}{|l|}{ Tetracyclic Antidepressants } \\
\hline Maprotiline & 19 & 57.9 & 4 & 0 & 211 \\
\hline Mirtazapine & 2,599 & 18.2 & 31 & 0 & 12 \\
\hline Lithium & 3,960 & 40.7 & 129 & 2 & 33 \\
\hline \multicolumn{6}{|l|}{ MAO Inhibitors } \\
\hline Phenelzine & 56 & 60.7 & 8 & 1 & 161 \\
\hline Tranylcypromine & 35 & 51.4 & 5 & 0 & 143 \\
\hline \multicolumn{6}{|l|}{ Other Antidepressants } \\
\hline Bupropion & 7,631 & 34.9 & 735 & 9 & 97 \\
\hline Nefazodone & 1,320 & 18.1 & 21 & 1 & 17 \\
\hline Trazodone & 12,538 & 18.8 & 140 & 0 & 11 \\
\hline \multicolumn{6}{|c|}{ Serotonin And Norepi Reuptake Inhibitors (SNRIs) } \\
\hline Venlafaxine & 5,510 & 21.8 & 136 & 12 & 27 \\
\hline Duloxetine & 36 & 22.2 & 0 & 0 & 0 \\
\hline \multicolumn{6}{|c|}{ Selective Serotonin Reuptake Inhibitors (SSRIs) } \\
\hline Citalopram & 5,251 & 16.3 & 141 & 3 & 27 \\
\hline Fluvoxamine & 366 & 19.4 & 7 & 1 & 22 \\
\hline Escitalopram & 3,008 & 13.0 & 17 & 0 & 6 \\
\hline Paroxetine & 9,258 & 9.4 & 40 & 2 & 5 \\
\hline Sertraline & 12,002 & 9.8 & 53 & 1 & 4 \\
\hline Fluoxetine & 7,008 & 8.1 & 28 & 1 & 4 \\
\hline
\end{tabular}




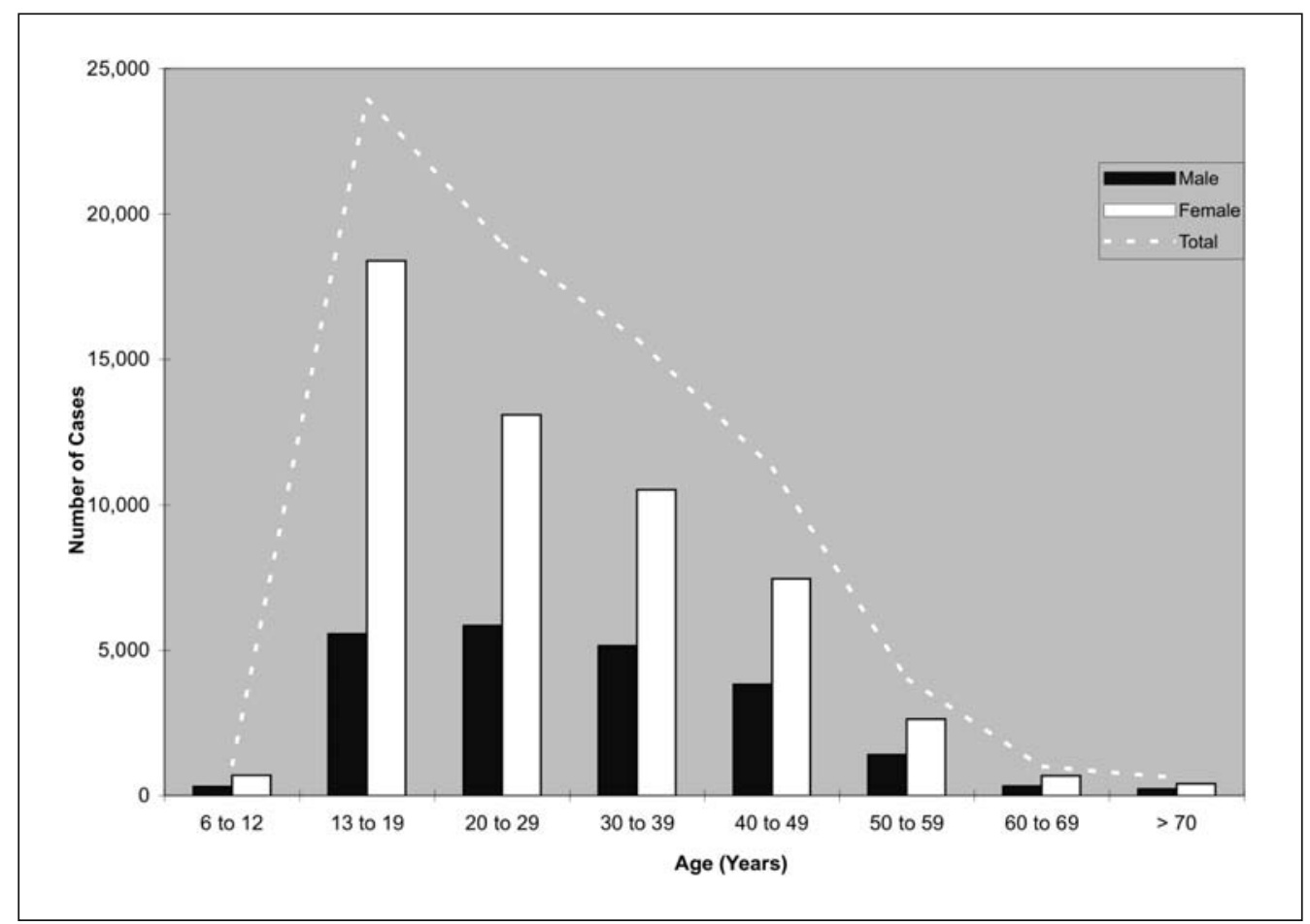

Figure 1: Age and gender distribution for suicidal antidepressant ingestions.

single-agent antidepressant ingestions. Of the single-agent antidepressant ingestions, 82,802 were coded with a specific, identifiable antidepressant approved for use in the US, formulated alone (without a benzodiazepine, phenothiazine or other antipsychotic), and formulated as an oral medication rather than as a topical cream. These latter 82,802 cases are the basis for this study.

The most commonly implicated antidepressants were trazodone (15.1\%), sertraline (14.5\%), paroxetine (11.2\%), amitriptyline (10.0\%), bupropion (9.2\%), fluoxetine (8.5\%), venlafaxine (6.7\%), and citalopram (6.3\%). Together these 8 antidepressants accounted for $81.5 \%$ of the cases.

The age distribution of the single-agent suicidal antidepressant ingestions (Figure 1) showed a peak in the teen years $(28.9 \%$ of cases) and a predominance of women (69.5\% of suicidal antidepressant ingestions). In contrast, the frequency of fatal cases peaked in the 40- to 49-year-old age group (Figure 2). Again, there were more female cases, accounting for $56.5 \%$ of fatal suicidal antidepressant ingestions. While females were more frequently implicated than males in both the fatal and nonfatal cases, the gender ratios were significantly different in nonfatal and fatal suicidal ingestions (chi square, $\mathrm{p}<0.001$ ), with less of a gender difference observed in fatal cases. The age distribution by antidepressant type (Figure 3), showed suicidal ingestions of the SSRIs, serotonin and norepinephrine reuptake inhibitors (SNRIs), and other antidepressants peaking in the teens, lithium in the twenties, the tricyclics and tetracyclics in the thirties, and MAO inhibitors in the forties.

\section{Hazard Index}

A large variation was seen in the severity of suicidal overdoses involving the different antidepressant types (Table 1). Of the 82,802 suicidal ingestions, there were 138 fatalities and 3,173 lifethreatening (major) outcomes; thus there were 40 major or fatal outcomes per 1000 study cases. Weighting each antidepressant type equally, the mean hazard index (rate of life-threatening or lethal outcomes per 1000 single-agent suicidal ingestions) for the 25 antidepressants analyzed was 79, with a range of 0 to 292. Amoxapine (292), maprotiline (211), and desipramine (187) had the highest hazard indices. All of the tricyclic antidepressants, MAO inhibitors, maprotiline, and bupropion were in the more severe half of antidepressants ranked by hazard index, with hazard indices $>55$. In contrast, all of the SSRIs, the combined serotonin and norepinephrine reuptake inhibitors, lithium, nefazodone, trazodone, and mirtazapine were ranked in the less severe half of antidepressant categories. All SSRIs had hazard indices $<28$, whereas all tricyclic antidepressants had hazard indices $>55$. The mean hazard index for TCAs was significantly higher than for SSRIs (T-test, $\mathrm{p}=0.0016$ ).

An inverse correlation was found between the hazard index and the exposure frequency $(\mathrm{R}=-0.423, \mathrm{p}=0.035)$. Thus in general, the more hazardous antidepressants were implicated in suicidal overdoses less frequently (Figure 4). Two notable outliers were observed, amitriptyline and bupropion, both with relatively high hazard indices and high overdose frequency. Table 1 and Figure 4 also demonstrate that the tricyclic antidepressants (and bupropion) 


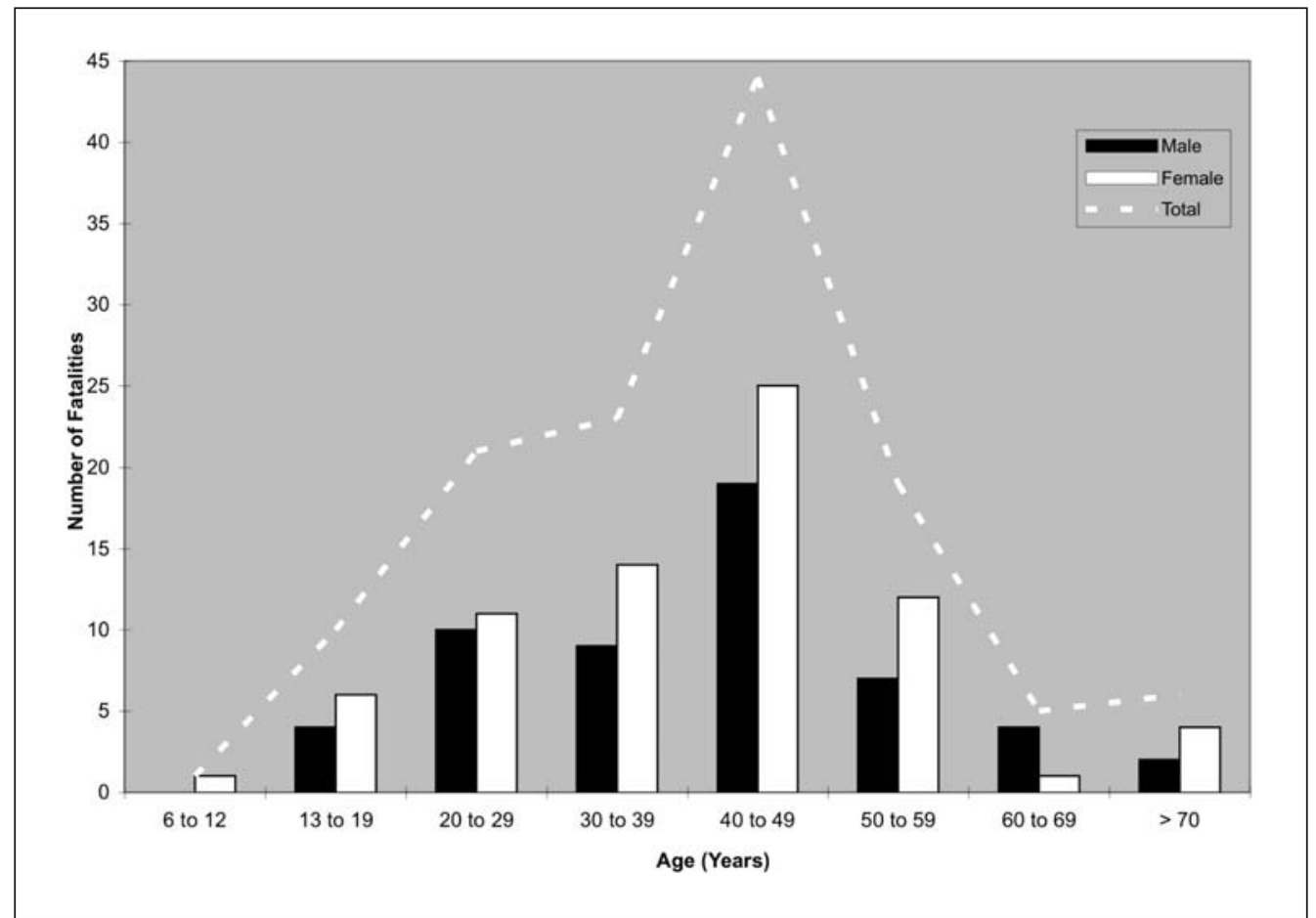

Figure 2: Age distribution for fatal suicidal antidepressant ingestions.

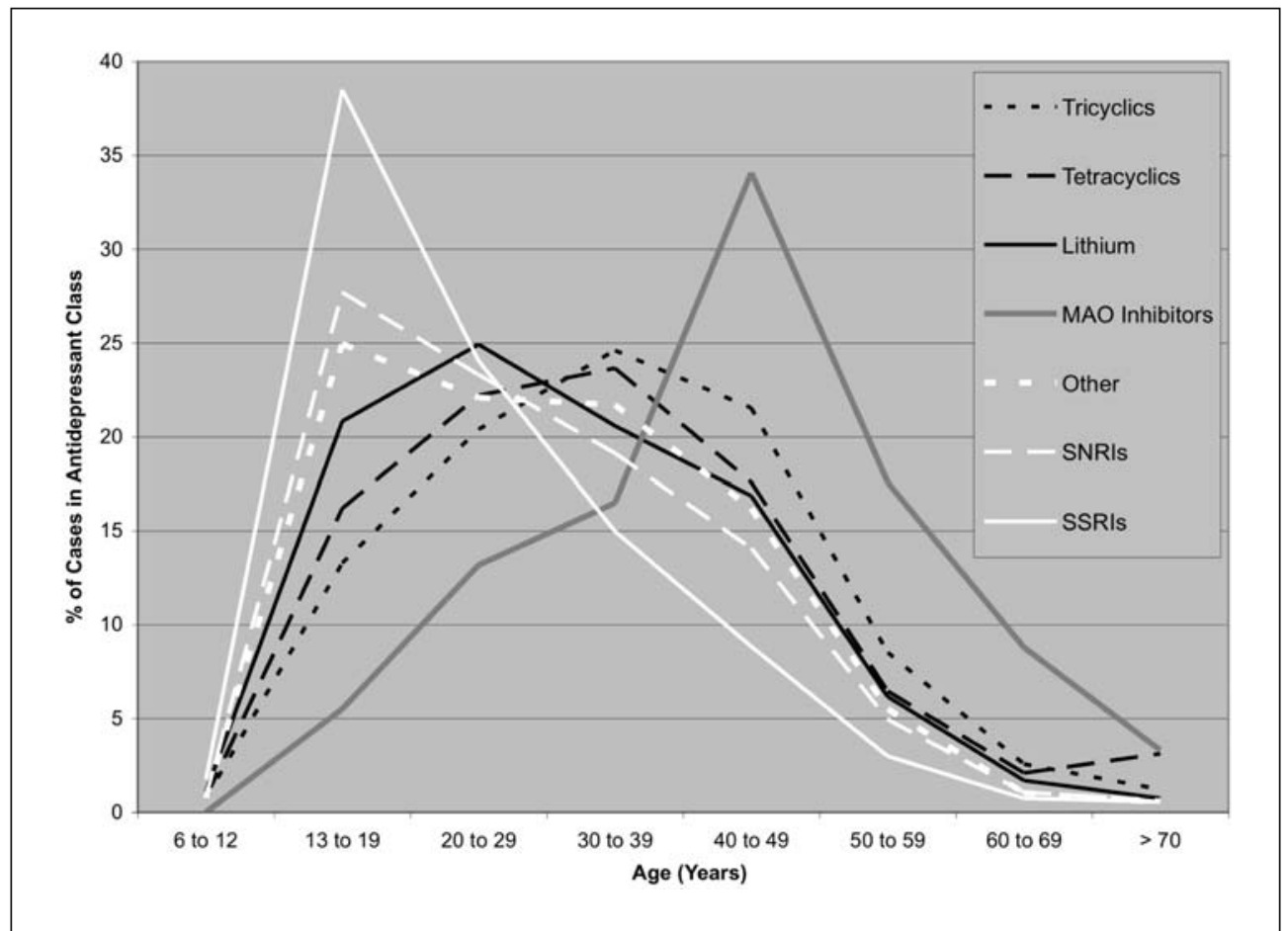

Figure 3: Antidepressant class by age group, suicidal ingestions. 


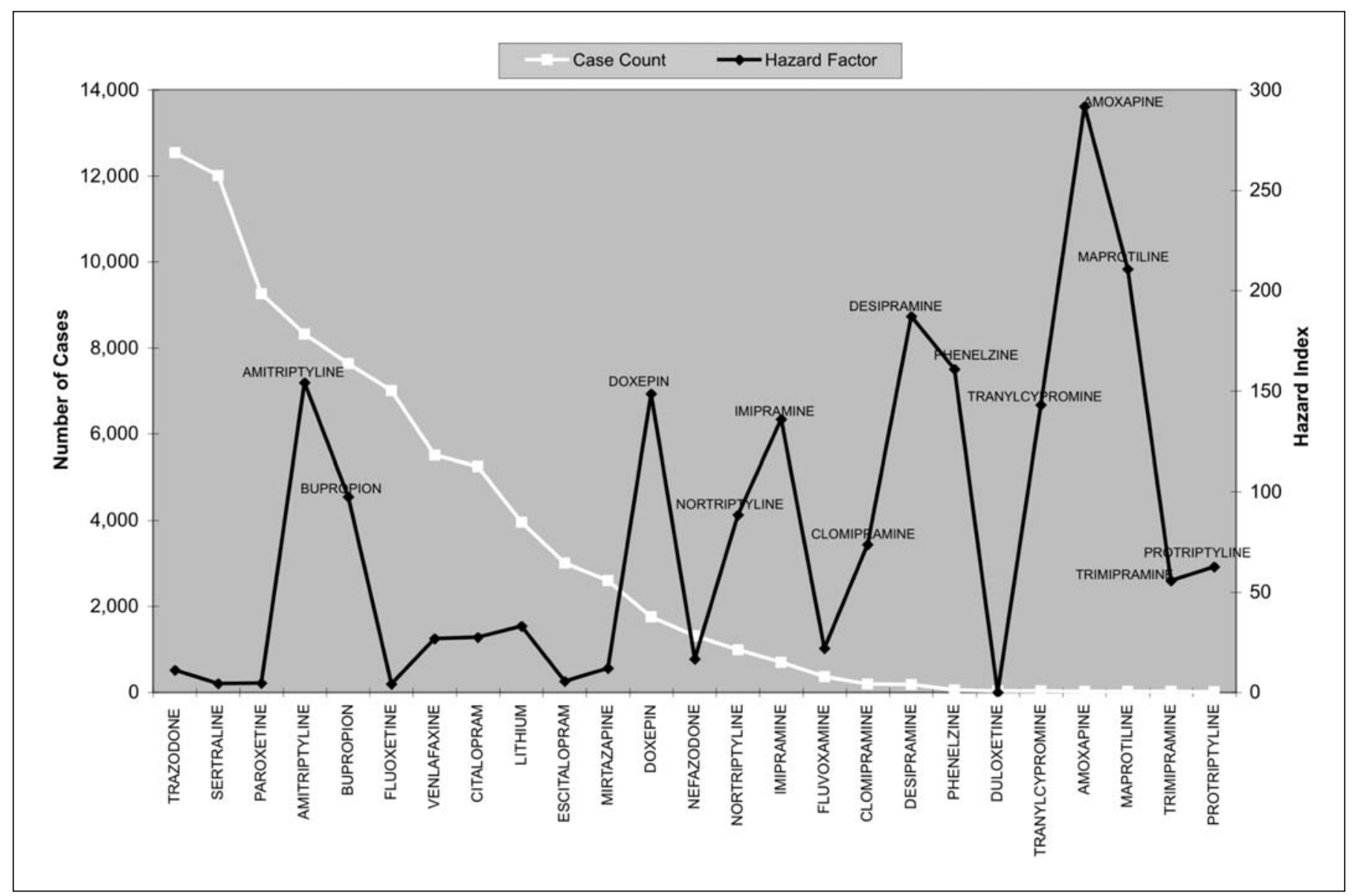

Figure 4: Number of cases versus hazard index by antidepressant type.

have the highest hazard indices, but the SSRIs and other antidepressants are more frequently implicated in suicidal ingestions.

Critical care treatment is another indicator of overdose severity. A greater percentage of the tricyclic antidepressant, MAO inhibitor, and maprotiline cases required critical care compared to the SSRI, SNRI, and other antidepressant cases (Table 1). A significant correlation was observed between the hazard index and use of critical care for the 25 antidepressant types $(R=0.797$, $p<0.001)$.

\section{CLINICAL EFFECTS}

A total of 85,734 clinical effects coded as "related" or "unknown if related" to the ingestion were observed in the 82,802 cases; some cases had no clinical effects and others had one or more clinical effects. The percent of patients who ingested each antidepressant type and experienced a given clinical effect is shown in Table 2. (For simplicity, only those effects that occurred with a frequency of at least $1.0 \%$, in overdose with at least 1 antidepressant type are listed.) To provide a visual profile rather than a statistical analysis of differences in clinical manifestations, shading is used to highlight cases with a relatively frequent occurrence of a given clinical effect. Dark gray shading shows the clinical effect frequency for the antidepressant type was one of the highest, or between $67 \%$ and $100 \%$ of the highest value observed for that clinical effect in any antidepressant type, 33-66\% for light gray shading, and no shading for the lowest frequencies.

Cardiac effects were seen most commonly in tricyclics, maprotiline, MAO inhibitors, and bupropion. Gastrointestinal manifestations were remarkably absent with TCAs compared to the SSRIs, SNRIs, and other antidepressants. Coma, confusion, lethargy, seizures, and agitation were prominent with TCA overdose. Even compared with the other tricyclics, amoxapine was distinguished by high rates of cardiovascular effects, multiple seizures and status epilepticus, renal failure, hyperventilation, respiratory depression or arrest, acidosis, and fever or hypothermia. Desipramine showed a similarly toxic profile with regard to seizures, conduction disturbance, and hypotension.

$\mathrm{MAO}$ inhibitor overdoses were associated with high frequencies of serious clinical effects in all symptom categories and notably high rates of hypertension and hypotension as well as hyperthermia. The two tetracyclics studied appear to differ in clinical effect profile. Maprotiline had substantially higher frequencies of nearly all clinical effects compared to mirtazapine. This supports the finding that maprotiline has a 17-fold higher hazard index than mirtazapine (Table 1). 


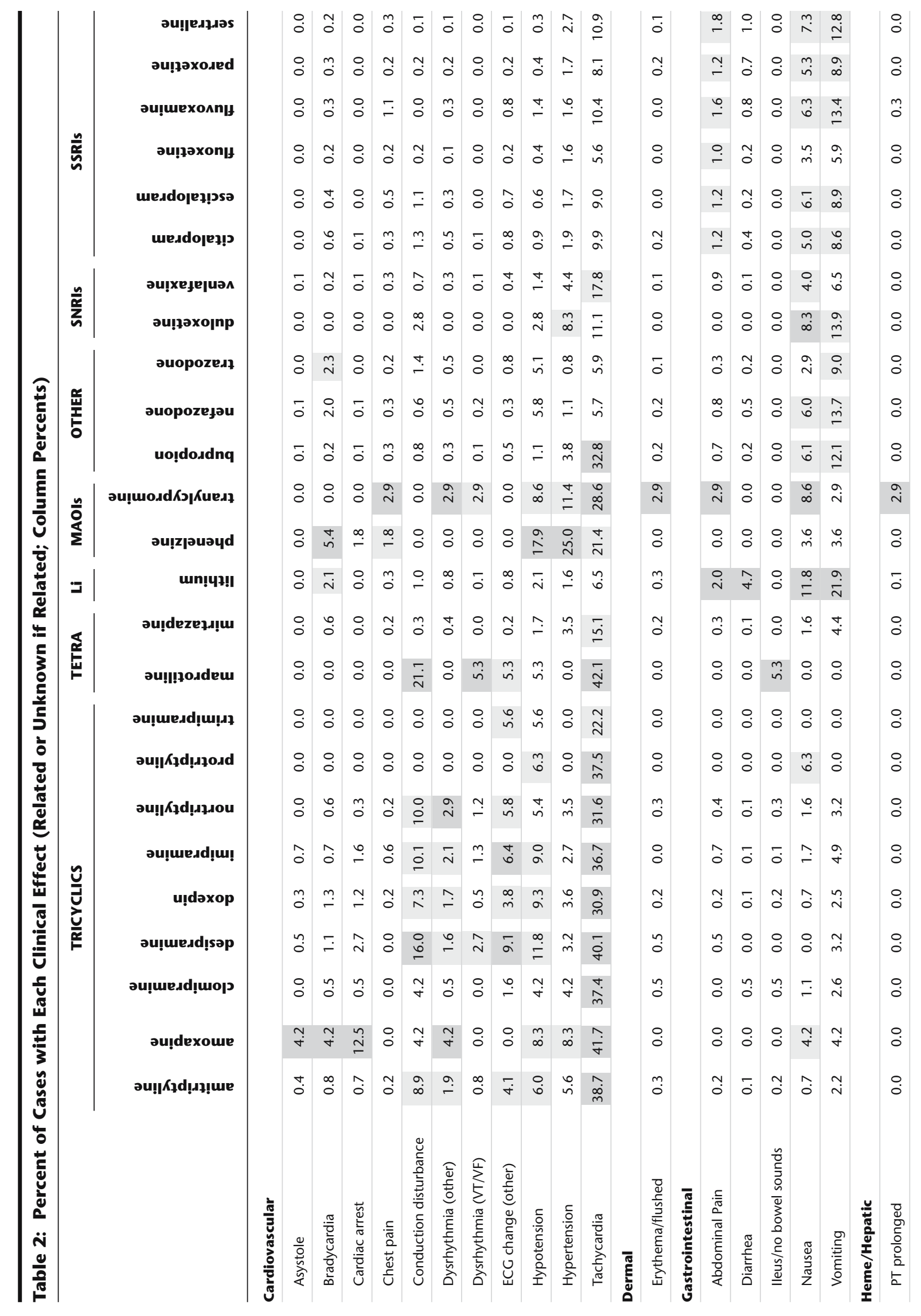




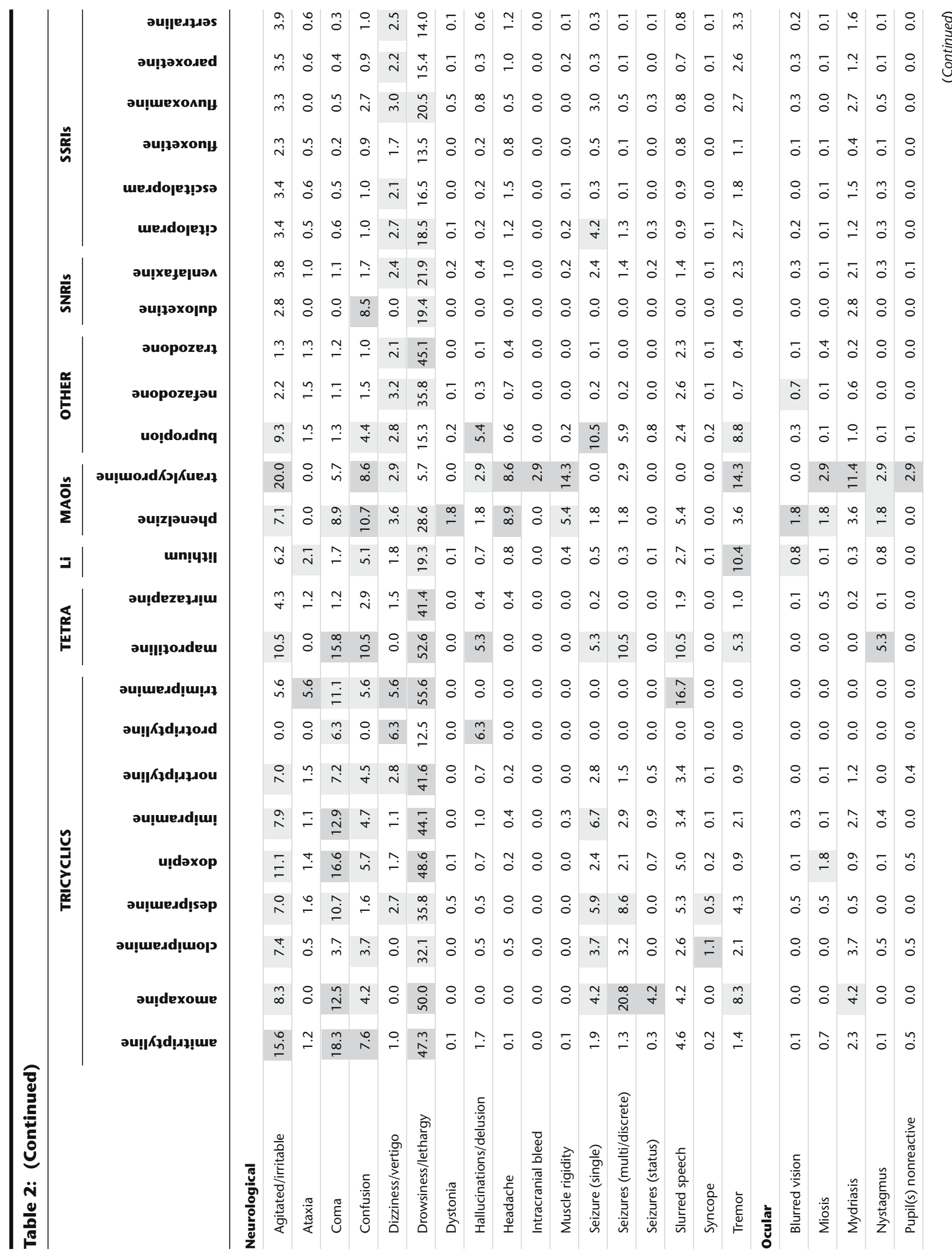




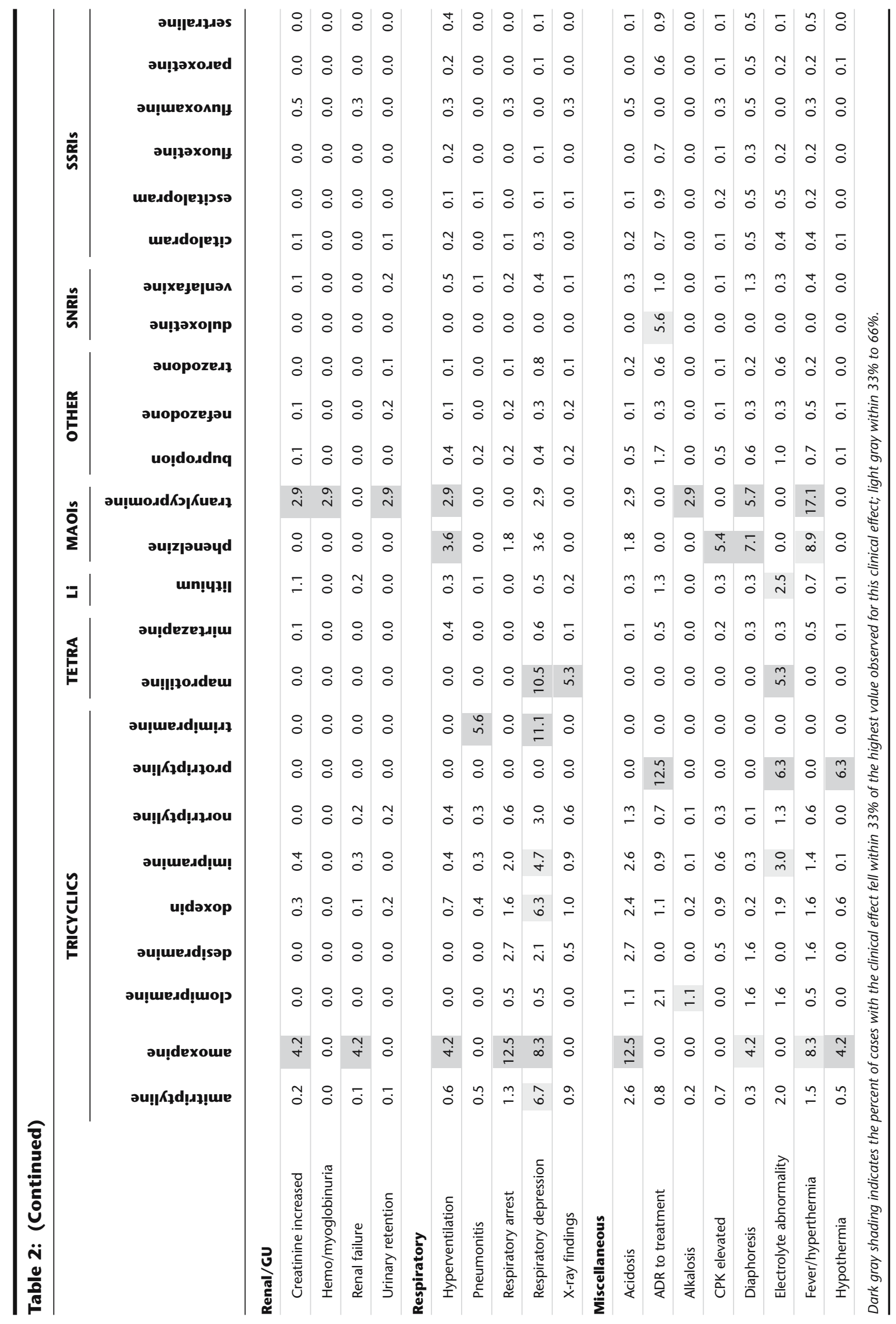


Bupropion showed the highest rate of single seizures and a high rate of hallucinations, with an otherwise relatively mild side-effect profile compared to the other antidepressants. Except for drowsiness, trazodone had a remarkably benign side-effect profile. Both SNRIs, venlafaxine and duloxetine, showed benign side-effect profiles predominantly limited to nausea, vomiting, and drowsiness.

SSRIs showed lower frequencies of cardiac, neurological, ocular, renal, and respiratory symptoms. Though relatively low in frequency compared with other antidepressants, gastrointestinal and neurological symptoms were the predominant clinical effects seen with SSRI overdose. These neurological effects were relatively benign, mostly limited to dizziness, drowsiness, and agitation. Of note, citalopram showed a single-seizure rate comparable to that of the tricyclics.

\section{DISCUSSION}

The age distribution of single-agent suicidal ingestions of antidepressants reported to TESS from 2000 to 2004 showed a peak in the teen years and a predominance of women (Figure 1). These data are consistent with the US rates of self-harm poisonings in 2003-not limited to antidepressants-as reported in WISQARS (Web-based Injury Statistics Query and Reporting System), in which the peak self-harm rate was observed in 15- to 19-year-olds [15]. WISQARS also reports a peak suicidal poisoning death rate in the 45- to 49-year-old age group in 2003 in the US, mirroring the peak in 40- to 49-year-olds observed in TESS for fatal antidepressant suicides (Figure 2).

This study demonstrated a shift in the peak age by antidepressant type, with SSRIs, SNRIs, and the group of other antidepressants peaking in teens, lithium in the twenties, tricyclics and tetracyclics in the thirties, and MAO inhibitors in the forties (Figure 3). Interestingly, suicidal overdoses of the newer antidepressant types had younger peak ages compared to antidepressants that had been marketed for decades (tricyclics, MAO inhibitors). This may have been a contributing factor in the rightward shift of the age distribution for fatal suicidal antidepressant ingestions compared to nonfatal cases (Figures 1 and 2), as there may be greater prescribing of more toxic and older antidepressants in older age groups.

A number of investigators have used hazard analyses to assess product safety using TESS data. Some investigations have included moderate effects in the numerator $[16,17]$ while others have limited the rate to major effects and deaths divided by total exposure count $[18,19,20]$. Sometimes the rate is normalized or converted to a rate ratio, taking the ratio of the rate for a specific product or class to the rate for all substances $[17,18,20]$. Normalization provides rapid assessment of outliers, as products with higher-thanaverage severity have hazard factors greater than 1.0. However, normalization was not used in this investigation as temporal shifts to more- or less-toxic antidepressant prescribing could affect the relative outcome, undermining comparisons of past and future data. In this analysis, the hazard index was defined to focus on the outcomes of greatest interest by including life-threatening and lethal cases in the numerator and all single-agent suicidal exposures to the antidepressant type in the denominator.

Whatever the methodology employed, prior hazard or toxicity comparisons have guided regulatory decisions or advocacy of product reformulation, repackaging, relabeling or recall $[17,18$, $21,22]$. This study used a hazard index and an analysis of associated clinical effects to update and extend the multiple previous comparisons of the relative toxicity of antidepressants in overdose with a large patient sample $(82,802$ cases) and large number of antidepressant types (25). By focusing on antidepressants approved and marketed in the US and by quantifying the relative rates of life-threatening or lethal outcomes, the results allow clinicians to make more-informed prescribing decisions, potentially decreasing the severity and cost of antidepressant overdoses.

This analysis of clinical effects associated with the various antidepressant types quantified the differences in clinical presentations and showed some unexpected as well as other well-recognized clinical profiles. Cardiovascular effects were common in suicidal TCA overdoses reported to TESS, including a high frequency of tachycardia, conduction disturbances, dysrhythmias, and hypotension. Neurological effects—coma, lethargy, and seizures-were also prominent. Maprotiline had a substantially higher frequency of nearly all clinical effects as compared to the other tetracyclic antidepressant mirtazapine, supporting maprotiline's 17-fold higher hazard index compared with mirtazapine.

Amoxapine and maprotiline had the highest hazard indices in this analysis, although they were implicated in very small numbers of cases (24 and 19, respectively), perhaps reflecting an adaptation to drug toxicity in overdose by prescribing physicians. A 1995 study from the UK corroborates the high fatality rate of amoxapine, reporting 157.18 deaths per million prescriptions of amoxapine, the highest rate, by 2-fold, of 25 antidepressants reviewed [23]. Our analysis of poison control data showed high rates of cardiovascular events, coma, status epilepticus, renal failure, respiratory depression, and acidosis with amoxapine.

In this analysis of the 2000-2004 TESS data, desipramine had the third highest hazard index but had a relatively low number of cases (187). Similarly, it had the second highest fatal toxicity index (deaths per million prescriptions) in a British analysis [23]. Desipramine also had the highest reported mortality index of 8 antidepressants studied by Rose using TESS data from 1989 to 1997, although notably, amoxapine and maprotiline were not included in that analysis [24]. Rose defined mortality index as the average death rate per year for patients presenting to a health-care facility with an overdose of the specific drug. Similarly, in an analysis of 1989 and 1990 TESS data combined with National Prescription Audit and Drug Abuse Warning Network data sets, of 5 antidepressants compared, desipramine had the highest relative risk of death in overdose-twice that of nortriptyline [25]. Compared to other TCAs, our study shows desipramine had a high rate of seizures, conduction disturbances, and hypotension.

In our analysis of TESS data, MAO inhibitors showed high frequencies of serious clinical effects in all clinical-effect categories. Lithium overdoses showed high rates of gastrointestinal effects, especially nausea and vomiting, as well as lethargy and tremor, 
despite a relatively low hazard index of 33, echoing findings from older (1989-1997) poison center data [24].

Previous analyses of TESS antidepressant data were limited to 5 or 8 antidepressant types $[24,25]$. Specific analyses of the newer antidepressant agents were impaired by the TESS generic coding system, which does not differentiate most of the individual SSRIs, SNRIs, or other newer antidepressants. Manual recategorization of nearly 1000 specific pharmaceutical products was required for this analysis. By recategorizing the suicidal antidepressant ingestions into 25 types, we were able to assess the two SNRIs, duloxetine and venlafaxine. Both had low hazard indices and relatively lower incidence of cardiovascular, neurological, and respiratory effects compared with the TCAs, tetracyclics, and MAO inhibitors. Since duloxetine was introduced just before the end of the study period, there were only a small number of cases reported (36); nonetheless, its hazard index was 0 , indicating no major or fatal outcomes. Although less symptomatic than most other antidepressant types, when clinical effects were present, duloxetine overdoses manifested hypertension, tachycardia, nausea, vomiting, confusion, and drowsiness.

Recategorization also enabled a comparative assessment of bupropion, trazodone, and nefazodone (comprising a group labeled "other" antidepressants). Bupropion showed the highest single-seizure rate of all antidepressants reviewed, and also high rates of hallucinations compared to other antidepressants. TESS data revealed a moderately high hazard index for bupropion, in the middle of the TCA range; however, this finding could not be compared with other studies since the bupropion fatality risk or hazard factor was not assessed by prior authors.

In TESS, the clinical effects for trazodone and nefazadone showed gastrointestinal effects and dizziness with frequency comparable to that of the SSRIs, but drowsiness at a frequency intermediate between the SSRIs and TCAs. Otherwise, the clinical-effect profiles for these 2 drugs were minimal, both having very low hazard indices in TESS. Trazodone also had a low index in 2 other fatality risk assessments, neither of which included nefazodone $[23,24]$.

Recategorization also allowed an analysis of each of 6 SSRIs. All had low hazard indices along with a relatively benign clinicaleffects profile. In general, the clinical-effect profile for the SSRIs was strikingly benign, with drowsiness as the primary manifestation. Gastrointestinal effects were the only symptoms that occurred more often in SSRI than TCA overdose. Of note, citalopram was the only SSRI with a high rate of single seizures in overdose, a finding that deserves additional investigation, as it has not been reported by others. Henry's prior investigation showed very low fatality indices for 4 SSRIs [23]. Likewise, Kapur cited Drug Abuse Warning Network data showing an 8-fold lower relative risk of death in overdose for fluoxetine compared to desipramine [25].

Studies from several countries suggest that fatality rates from suicidal overdoses vary considerably among available antidepressants. The older TCAs appear consistently more lethal in overdose than their newer counterparts. In particular, desipramine was associated with a roughly 2 -fold higher rate of deaths per overdose than other tricyclics [25], more than 16-fold greater than trazodone, and 8-fold greater than fluoxetine. Amitriptyline and dothiepin, the latter never approved for use in the US, were more likely to be associated with death in overdose than other widely used antidepressants in the UK $[11,26]$. One study conducted in the UK from 1987 to 1992, found 30.1 deaths per million prescriptions for all antidepressants [23], with 34.14 deaths per million prescriptions for TCAs, 13.48 for MAO inhibitors, and 2.02 for SSRIs. Even though prescribing rates were low, 2 TCAs had unusually high rates: amoxapine (157.18) and desipramine (75.76). The more toxic tricyclic antidepressants are more likely to lead to medical complications and admissions to intensive care units [23]. The most lethal antidepressants also demonstrate significant cardiovascular effects; tricyclic antidepressant poisoning leads to cardiovascular complications due to direct cardiotoxicity, CNS sedation, and respiratory suppression [25]. Newer antidepressants do not show this extreme direct cardiotoxicity.

While some may postulate higher death rates due to variations in antidepressant efficacy or even a propensity of an individual drug to actually promote suicidal behaviors [2], the most persuasive argument for the higher rates of mortality and higher hazard indices seen in overdose of selected antidepressants is the higher inherent toxicity of the antidepressant. Our data compare outcomes for suicide attempts, thus eliminating the variation in suicidal behavior frequency as a contributing factor. In contrast, the use of total prescriptions as the denominator for a mortality index is more subject to variation introduced by the efficacy of the drug in controlling or promoting suicidal ideation.

The importance of providing the practicing clinician with a hazard index for antidepressants is underscored by the reduced fatality rate observed in Sweden when prescribing patterns shifted. The increased use of antidepressants following the introduction of SSRIs coincided with a significant decline in fatal overdose rate for antidepressants as a class [27]. Between 1992 and 1994, prescribing of SSRIs doubled the use of antidepressants, yet there was a $12 \%$ reduction in the fatality rate from antidepressant overdose.

In addition to the improved outcomes expected following suicidal overdoses of less toxic antidepressants, decreased healthcare costs are a potential but unproven secondary benefit. One study in England and Wales compared hospital costs of overdoses with SSRIs and TCAs. Those taking SSRIs had a significantly shorter and less costly hospital stay, largely due to less utilization of intensive care units [28], but this savings was mitigated by the even greater cost of prescribing SSRIs compared to TCAs.

These results are subject to a number of limitations. Prescription rates were not available to establish therapeutic usage frequency. In addition, TESS data are subject to the limitations of a voluntary telephone reporting system, thus include less comprehensive clinical and laboratory data than those gathered in a clinical investigation based in a healthcare facility. Poison control data underrepresent deaths occurring at home or en route to the hospital and deaths not suspected as related to a poisoning or suicide. One author showed only about $12 \%$ of overall poisoning fatalities were reported to one state's poison center [29], although 
$53 \%$ of in-hospital poisoning deaths were reported [30]. Nonfatal suicidal overdoses are undoubtedly also underrepresented. However there is no evidence that underreporting of fatal or nonfatal suicidal poisonings would vary selectively from one antidepressant type to another.

The TESS data showed a large variation in the severity of suicidal overdoses by antidepressant type. Cyclic antidepressants, maprotiline, and MAO inhibitors had the highest rates of lifethreatening and lethal outcomes, while SSRI overdoses were uniformly less severe. The validity of the hazard index as a measure of overdose severity is corroborated by the finding that critical-care utilization correlated significantly with the hazard index. Remarkably, an inverse correlation was found between hazard index and exposure frequency, suggesting that-with some exceptions-physician prescribing is influenced by concerns about providing toxic substances to suicidal patients.

The authors have no potential financial conflicts of interest to report.

\section{REFERENCES}

1. Dumais A, Lesage AD, Alda M, Rouleau G, Dumont M, Chawky N, et al. Risk factors for suicide completion in major depression: a case-control study of impulsive and aggressive behaviors in men. Am J Psychiatry 2005;162 (11):2116-2124.

2. Friedman RA, Leon AC. Expanding the black box: depression, antidepressants, and the risk of suicide. $N$ Engl J Med 2007;356 (23):2343-2346.

3. Gould MS, Greenberg T, Velting DM, Shaffer D. Youth suicide risk and preventive interventions: a review of the past 10 years. J Am Acad Child Adolesc Psychiatry 2003;42(4):386-405.

4. Amos R, Appleby L, Kiernan K. Changes in rates of suicide by car exhaust asphyxiation in England and Wales. Psychol Med 2001;31(5):935-939.

5. Gunnell D, Middleton N, Frankel S. Method availability and the prevention of suicide: a re-analysis of secular trends in England and Wales 1950-1975. Soc Psychiatry Psychiatr Epidemiol 2000;35(10):437-443.

6. Lester D. Effects of detoxification of domestic gas on suicide in the Netherlands. Psychol Rep 1991;68(1):202.

7. Ohberg A, Lonnqvist J, Sarna S, Vuori E, Penttila A. Trends and availability of suicide methods in Finland: proposals for restrictive measures. Br J Psychiatry 1995;166 (1):35-43.

8. Wiedenmann A, Weyerer S. The impact of availability, attraction and lethality of suicide methods on suicide rates in Germany. Acta Psychiatr Scand 1993;88(5):364-368.

9. Carrington PJ, Moyer S. Gun control and suicide in Ontario. Am J Psychiatry 1994;151(4):606-608.

10. Kendell RE. Catalytic converters and prevention of suicides. Lancet 1998;352(9139):1525.

11. Farmer RD, Pinder RM. Why do fatal overdose rates vary between antidepressants? Acta Psychiatr Scand Suppl 1989;354:25-35.
12. Watson WA, Litovitz TL, Rodgers GC, Klein-Schwartz W, Reid N, Youniss J, et al. 2004 annual report of the American Association of Poison Control Centers Toxic Exposure Surveillance System. Am J Emerg Med 2005;23(5):589-666.

13. Litovitz T. The TESS database: Use in product safety assessment. Drug Saf 1998;18(1):9-19.

14. Uitenbroek DG [webpage on the Internet]. SISA Binomial [published 1997; cited 2006 Feb 25]. Available from:

http://home.clara.net/sisa/binomial.htm

15. Centers for Disease Control and Prevention, National Center for Injury Prevention and Control [webpage on the Internet]. Web-based Injury Statistics Query and Reporting System (WISQARS) [published 2005; cited 2006 Feb 27]. Available from: $w w w . c d c . g o v / n c i p c / w i s q a r s$

16. Litovitz T, White NC, Watson WA. Epidemiology of pediatric poison exposures: an analysis of 2003 poison control center data. Clin Ped Emerg Med 2005;6(2):68-75.

17. Woolf AD, Watson WA, Smolinske S, Litovitz T. The severity of toxic reactions to ephedra: comparisons to other botanical products and national trends from 1993-2002. Clin Toxicol 2005;43(5):347-355.

18. Litovitz T, Manoguerra A. Comparison of pediatric poisoning hazards: an analysis of 3.8 million exposure incidents. Pediatrics 1992;89(6):999-1006.

19. Cobaugh DJ, Krenzelok EP. Adverse drug reactions and therapeutic errors in older adults: a hazard factor analysis of poison center data. Am J Health Syst Pharm 2006;63(22): 222-2234

20. Crouch BI, Caravati EM, Mitchell A, Martin AC. Poisoning in older adults: a 5-year experience of US poison control centers. Ann Pharmacother 2004;38(12):2005-2011.

21. Tenenbein M. Unit-dose packaging of iron supplements and reduction of iron poisoning in young children. Arch Pediatr Adolesc Med 2005;159(6):557-560.

22. U.S. Department of Health and Human Services [webpage on the Internet]. News release: FDA announces plans to prohibit sales of dietary supplements containing ephedra (2003, December) [published 2003 Dec; cited 2005 Nov 4]. Available from: http://www.cfsan.fda.gov/ lrd/hhsephe2.htmlREF

23. Henry JA, Alexander CA, Sener EK. Relative mortality from overdose of antidepressants. BMJ 1995;310(6974):221-224.

24. Rose JC, Unis AS. A mortality index for postmarketing surveillance of new medications. Am J Emerg Med 2000;18(2):176-179.

25. Kapur S, Mieczkowski T, Mann JJ. Antidepressant medications and the relative risk of suicide attempt and suicide. JAMA 1992;268(24):3441-3445.

26. Montgomery SA, Baldwin D, Green M. Why do amitriptyline and dothiepin appear to be so dangerous in overdose? Acta Psychiatr Scand Suppl 1989;354:47-53.

27. Isacsson $G$, Bergman U. Risks with citalopram in perspective. Lancet 1996;348(9033):1033.

28. Ramchandani P, Murray B, Hawton K, House A. Deliberate self poisoning with antidepressant drugs: a comparison of the relative hospital costs of cases of overdose of tricyclics with 
those of selective-serotonin reuptake inhibitors. J Affect Disord 2000;60(2):97-100.

29. Linakis JG, Frederick KA. Poisoning deaths not reported to the regional poison control center. Ann Emerg Med 1993;22(12):1822-1828.

30. Soslow AR, Woolf AD. Reliability of data sources for poisoning deaths in Massachusetts. Am J Emerg Med 1992;10(2):124-127.

\section{APPENDIX A: DISCLOSURE STATEMENT OF THE AMERICAN ASSOCIATION OF POISON CONTROL CENTERS}

The American Association of Poison Control Centers (AAPCC, $w w w . a a p c c . o r g)$ maintains the national database of information logged by the 61 US poison control centers. Case records in this database are from self-reported calls; they reflect only information provided when the public or health-care professionals report an actual or potential exposure to a substance (e.g., an ingestion, inhalation, or topical exposure), or request information. Exposures do not necessarily represent a poisoning or overdose. The AAPCC is not able to completely verify the accuracy of every report made to member centers. Additional exposures may go unreported to poison control centers and data referenced from the AAPCC should not be construed to represent the complete incidence of national exposures to any substance(s).

Rev. January 2006.

Publication of these data was approved by the AAPCC Board of Directors. 